\title{
INTENTIONAL RESPONSES TO KINESTHETIC STIMULI IN OROFACIAL MUSCLES: IMPLICATIONS FOR THE COORDINATION OF SPEECH MOVEMENTS ${ }^{1}$
}

\author{
KELLY J. COLE ${ }^{2}$ AND JAMES H. ABBS \\ Speech Motor Control Laboratories, Waisman Center, University of Wisconsin-Madison, Madison, Wisconsin 53706
}

Received February 28, 1983; Revised June 20, 1983; Accepted August 2, 1983

\begin{abstract}
Reaction times (RTs) from human orofacial muscles were obtained with labial displacement and auditory stimuli to evaluate the potential for contributions by these sensorimotor processes to speech movement coordination. Because speech requires both concurrent and sequential movements, intramovement (labial stimulus, labial response) and intermovement (labial stimulus, lingual response) RT minima were examined.

Intramovement and intermovement RT minima were from 46 to $52 \mathrm{msec}$. Response-related reductions in background muscle activity were observed with latencies as short as $35 \mathrm{msec}$. These observations indicate a potentially important role for $\mathrm{RT}$ processes in the coordination of speech movements. These control processes would appear to be open-loop and predictive.
\end{abstract}

A classical question in the study of motor behavior has been whether afferent signals produced by one movement contribute to the temporal-spatial patterning in concurrent or subsequent movements (e.g., Weiss, 1941; Lashley, 1951; Evarts et al., 1971). Whereas Lashley's (1951) seminal paper focused on afferent contributions to the sequencing of serial motor actions, the broader issue of temporal-spatial patterning among multiple movements has also been of interest, especially in its relevance to speech motor behavior (Kozhevnikov and Chistovich, 1965; MacNeilage, 1970; Ohala, 1970; Kent and Moll, 1975; Abbs and Cole, 1982). Neural control of the quasiconcurrent movements of the upper and lower lips, mandible, tongue, velopharynx, pharynx, larynx, and respiratory system constitutes an extraordinary motor coordination achievement; indeed, speech reflects coordination par excellence. Understanding the control of complex, coordinated limb movements thus should be enhanced by considering the role of sensorimotor actions in speech.

In recent years, evidence has emerged indicating that afferent-dependent processes are involved in speech movement coordination. These data include demonstra-

\footnotetext{
${ }^{1}$ This work was supported by Program Grant NS 13274-07 from the National Institute of Neurological and Communicative Disorders and Stroke, and Core Support Grant 5-P30-HD-003352-14 from the National Institute of Child Health and Human Development. Abstracts of these experiments have been published elsewhere (Cole and Abbs 1979, 1981).

2 To whom correspondence should be addressed.
}

tions that complex speech motor objectives are achieved over multiple repetitions with significant covariance among the constituent submovements (Hixon et al., 1973; Hasegawa et al., 1976; Hughes and Abbs, 1976; Hunker and Abbs, 1982). Consistent with these observations are reports of short-latency compensatory responses in one speech movement following the unexpected disturbance of a synergistic movement (Folkins and Abbs, 1975, 1976; Abbs and Gracco, 1982; Gracco and Abbs, 1982a, b; Kelso et al., 1982). For example, when the jaw was interfered with during speech, compensatory responses in the lips were critical for the achievement of coordinated lip-jaw goals (Folkins and Abbs, 1975, 1976; Kelso et al., 1982). Likewise, interference with jaw movement during a coordinated jaw-tongue speech goal yielded compensatory adjustments in tongue muscles (Kelso et al., 1982). Comparable compensations were reported in studies in which speech movements of the upper or lower lip were disturbed through unanticipated loading (Abbs and Gracco, 1982; Gracco and Abbs, $1982 \mathrm{a}, \mathrm{b})$. Muscle responses with latencies from $25 \mathrm{msec}$ to more than $80 \mathrm{msec}$ have been observed in these studies.

In considering the afferent-dependent nervous system mechanisms that might contribute to speech movement coordination, reaction time (RT) processes that are ingrained into the motor repertoire through learning are appealing possibilities, especially in view of their adaptive capabilities. Although RTs have often been discounted as being too slow for contributions to the rapid movement sequences of speech (Lashley, 1951; Kozhev- 
nikov and Chistovich, 1965; Kent, 1976), recently reported RT minima suggest a reassessment of this position. Voluntary limb muscle responses with latencies from 40 to $65 \mathrm{msec}$ have been reported for kinesthetic stimuli delivered to a body region distant from the responding limb (Wyrick and Duncan, 1974; Hufschmidt et al., 1977; Lansing and Meyerink, 1981). Tongue muscle RTs to lingual stimuli (unspecified) have been reported with latencies of $40 \mathrm{msec}$ (Linke, 1977). Additionally, for rapid voluntary movements, suppression of background EMG in antagonist muscles has been reported as the earliest indication of a limb RT response, with latencies of 40 to 50 msec (Hufschmidt and Hufschmidt, 1954; Luschei et al., 1967). A sensorimotor process with response latencies as short as those indicated by these RT data may be capable of contributions to the compensatory responses following load disturbances of speech movements, and to speech movement coordination (Abbs and Cole, 1982).

To assess whether RT processes have latencies that could permit their contributions to the coordination of spcech movements, it was the goal of this study to obtain an empirical estimate of minimal latencies for voluntary orofacial muscle responses, using a labial displacement stimulus. Of particular interest were the latency capabilities for intermovement sensorimotor actions; namely, R'I responses in an orofacial structure to stimuli applied to a separate orofacial region.

Three separate experiments were conducted. Using a labial displacement stimulus, intramovement (labial response) RTs were obtained in experiment 1 and intermovement (lingual response) RTs were obtained in experiment 2. In experiment 3, labial RTs to an auditory stimulus were obtained in view of the potential significance of auditory information to speech control and in light of data from individual subjects implying shortlatency RT minima to auditory stimuli (Luschei et al., 1967; McFarlane, 1975). Experimental conditions optimal for rapid RT production were incorporated by: (1) employing a simple (no choice) RT paradigm; (2) using well learned (speech) responses; and (3) providing extensive practice on the task.

\section{Materials and Methods}

Subjects. Four normal male subjects from 18 to 23 years of age participated in experiment 1 (labial stimulus, labial response) and experiment 3 (auditory stimulus, labial response). Stimulus conditions were counterbalanced for order; subject 1 (S1) and S3 began with the auditory stimulus, whereas $\mathrm{S} 2$ and $\mathrm{S} 4$ began with the displacement stimulus. In experiment 2, the subjects were four females, from 23 to 33 years of age. All subjects were paid for participation in the experiments.

Apparatus. The subject was seated in a dental chair, slightly reclined, and the room was quiet and dark. The subject's head was supported in a U-shaped cradle that restricted lateral head movement. For the labial displacement stimulus, a hook that was shaped to fit in the corner of the mouth was coupled to a standard push-type solenoid; the hook was oriented so as to pull posteriorly and laterally on the right corner of the mouth when the solenoid was activated. A sustained prestretch of the oral angle of 10 to $15 \mathrm{gm}$ was maintained for all three experiments. The displacement stimulus used in experiments 1 and 2 was superimposed upon the sustained prestretch, with a rise time of $10 \mathrm{msec}$ and a peak displacement of 7 $\mathrm{mm}$ that had a duration of $25 \mathrm{msec}$. The displacement and static force applied to the oral angle were monitored, using strain gauges bonded on the hook and linkage assemblies. Encasement of the mechanical stimulus generator in sound-absorbing material and a lead box rendered it inaudible to the subject during the experiment. For experiment 3 , the auditory stimulus was produced by an electronic signal generator (Interstate F47) which delivered a single cycle of a $4-\mathrm{kHz}$ sinusoid when triggered. This signal was amplified and transduced through a loudspeaker placed directly behind the subject at ear level. The acoustically measured waveform was $450 \mu \mathrm{sec}$ in duration with a peak sound pressure level of $101 \mathrm{~dB}$ $\left(0.0002\right.$ dynes $\left./ \mathrm{cm}^{2}\right)$ that occurred at $2.5 \mathrm{kHz}$ (as measured with an impulse sound level meter with $1 / 3$ octave filtering). This stimulus was perceived as a comfortably loud click.

Labial myoelectric activity from the orbicularis oris inferior (OOI) muscle was sensed for experiments 1 and 3 , using miniature silver/silver chloride surface electrodes in a bipolar configuration (Cole et al., 1983). The electrodes were placed just inferior to the vermillion border, 3 to $4 \mathrm{~mm}$ apart, and centered over the left side of the lower lip. All placements yielded phasic activity for lip elevation gestures that was consistent with EMG expected for OOI. For experiment 2, activity from the tongue muscle superior longitudinus (SL) was sensed with 70- $\mu \mathrm{m}$ enamel-coated copper fine-wire electrodes. Each hooked wire was inserted individually with a hypodermic needle through the superior surface of the tongue, about 4 and $8 \mathrm{~mm}$ from the tongue tip for each electrode, respectively. For all subjects in experiment 2, phasic tongue muscle activity was associated with lingual elevation for a "d" sound. A standard pediatric EKG electrode was placed on the subject's forehead to serve as the reference electrode.

The EMG signals were amplified and filtered to yield a passband of 125 to $2500 \mathrm{~Hz}$. EMG and stimulus displacement signals were digitized on-line at 4000 samples/ sec/channel by a PDP-12 computer and stored for analysis. For experiment 3 , the pulse which triggered the auditory stimulus signal generator was recorded instead of the stimulus' acoustic waveform. A 2.8-msec delay between onset of this voltage step and onset of the acoustic waveform was accounted for in the latency measurement procedures. All data signals were recorded on FM tape.

Experimental procedures. When in position for the experiment, the subjects were told that their reaction time was being tested. They were instructed to close their eyes and produce the sound " $a$ " as if they were speaking to someone about 10 feet away. The experimenter monitored EMG (either OOI or SL), and the subjects were instructed, as necessary, to maintain quiescent $\mathrm{EMG}$ levels during this phonation. Upon detecting a stimulus, subjects were instructed to "quickly and forcefully" produce a " $b$ " sound (for experiments 1 and 3) or a " $\mathrm{d}$ " sound (for experiment 2). After each response, they were 
to immediately resume the "ah" phonation and their vigilant, response-ready state. In this manner it was possible to elicit several responses during each of the sustained phonation trials. Subjects initiated and terminated each phonation trial at will; phonation was generally sustained for 4 to $5 \mathrm{sec}$. Although rapid responses were emphasized, subjects were cautioned against anticipating the stimuli and producing false responses. For catch trials, stimuli were either withheld entirely, or withheld after a single stimulus was delivered. These catch trials were selected randomly with a ratio of experimental-to-catch trials of 15 .

At the start of phonation, the experimenter initiated a computer program which selected an interstimulus interval (ISI) value sequentially from a randomly generated table with 95 entries. This table was generated from a pool of 11 values that ranged from $0.5 \mathrm{sec}$ to 3.0 sec, at 0.25 -sec increments. These values were used to minimize stimulus anticipation (Baumeister and Joubert, 1969; Schupp and Schlier, 1972). The same ISI table was used for all experimental sessions. After the ISI duration had elapsed, the computer triggered the stimulus device and initiated analogue-to-digital conversion for $254 \mathrm{msec}$. The computer repeated the process by advancing to the next value in the ISI table, unless stopped by the experimenter. As a result of the fixed data sampling period, the actual ISI equaled the computer-tabled ISI plus $254 \mathrm{msec}$.

At the beginning of each session. EMG data were recorded for 25 stimulus presentations with subjects instructed not to respond; these data served as a basis for detecting possible electrical artifact, electrode motion artifact, and reflex or startle response contamination. Next, 25 productions of the speech response without the stimulus were recorded. After these first 50 pretrials, between 300 and 420 RT responses were obtained from all subjects but one (S3, experiment $2 ; 181$ responses). At the end of the session, the 50 "do not respond" and spontaneous response trials that began the session were repeated.

$R T$ measurement. RTs were measured interactively with a computer graphics display of each digitized EMG response and the accompanying stimulus. Both SL and OOI were generally quiescent during "ah" phonation, making the onset of phasic muscle activity unambiguous. Subsets of the data were remeasured, yielding an error range of 3 to $5 \mathrm{msec}$. Records for which phasic muscle activity onset could not be discerned easily were few, and these were eliminated. Some of these records were instances of false response, as verified by analysis of the accompanying speech acoustic signal.

\section{Results}

Experiment 1: Labial stimulus, labial response. Myoelectric data were carefully evaluated for the presence of startle or reflexive responses. Inspection of EMG in the "do not respond" condition revealed no evidence of such contamination (Fig. 1). EMG records from the response condition were examined as well for reflex or startle responses that could obscure the onset of voluntary muscle activity. No evidence of reflex or startle responses was observed during these response conditions (Fig. 2).

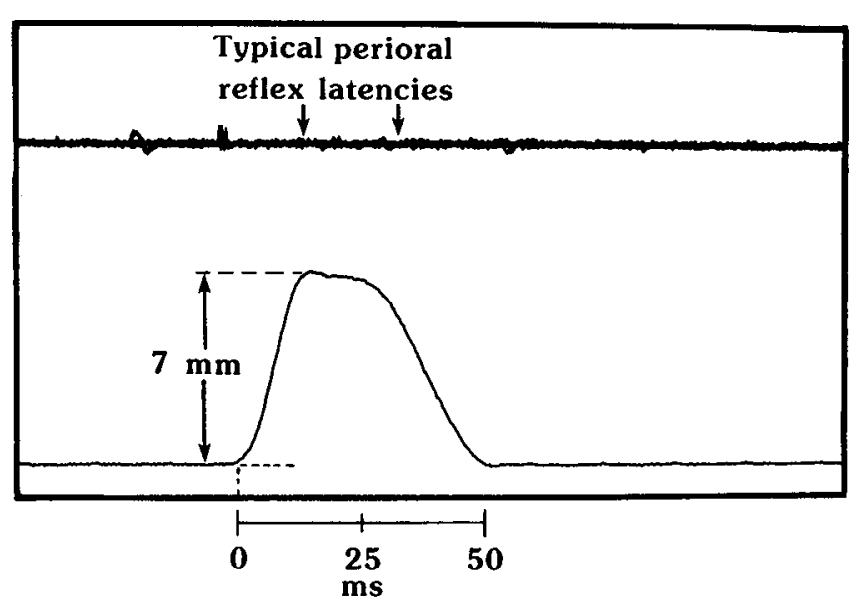

Figure 1. An oscillographic record of the displacement stimulus used in experiments 1 and 2, along with 10 superimposed records of OOI EMG from a subject who was instructed not to respond to the displacement stimulus while prodcing a sustained "ah" sound. The EMG records were aligned with the onset of stimulus displacement. The arrows indicate typical perioral reflex latencies of 15 and $35 \mathrm{msec}$ for the ipsilateral and contralateral reflex components, respectively.

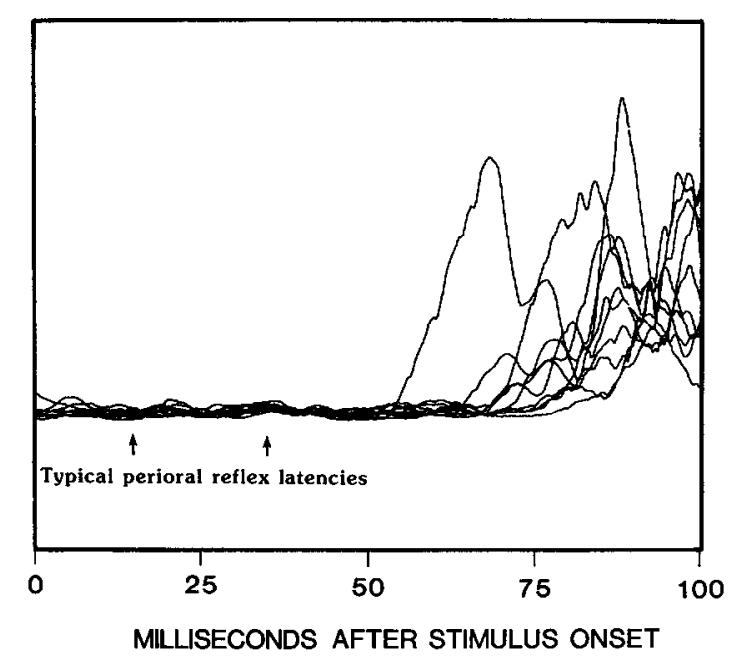

Figure 2. 'I'en superimposed records of OOI EMG, fullwave rectified and smoothed (time constant of $5 \mathrm{msec}$ ), from a single subject in the RT response condition. Typical perioral reflex latencies are indicated as in Figure 1.

The absence of reflex responses, along with the relative muscle quiescence during phonation, made discrimination of intended muscle onset unambiguous.

The sensitivity of RT minima to even a single spurious value warranted treatment of the data in a manner to ensure against artificially low estimates from responses not of stimulus-elicited origin. In this regard, false starts, defined here as completed or aborted responses occurring prior to stimulus onset, occurred very infrequently. For all subjects in experiment 1 , false starts averaged less than three per session, which was $0.6 \%$ of the response total. Except for the first foil trials for a few subjects, no false starts occurred during foil conditions. The subjects' estimates of false starts agreed with these observations. Also, only $0.3 \%$ of the responses began earlier than 40 
msec poststimulus, further indicating that subjects waited for stimulus onset. Nevertheless, a procedure to eliminate outliers from each subject's distribution of RTs was employed. ${ }^{3}$ For experiment 1 , only 7 values were eliminated out of 1449 responses. One eliminated response was greater than $40 \mathrm{msec}$, and thus the procedure only slightly sharpened the left tails of the already well defined response latency histograms.

A response latency histogram of the OOI activation RTs that remained after the treatment discussed above is shown for each subject in Figure 3 (unshaded distributions). For S4, an equipment malfunction resulted in his being in position for the experiment for well over an hour before the session could begin. The response latencies for $\mathrm{S} 4$ were much slower than for any other subject, supporting S4's claim of fatigue. For this reason, the data shown in Figure 3 for S4 were obtained in a subsequent experimental session on another day.

Given the precautions and controls noted previously, a valid and reliable estimate of the RT minimum for each subject in this experiment probably is within the fastest $25 \%$ of the responses, that is, the range of RTs from their absolute minimum to the first quartile (Q1) value. However, because each histogram comprises approximately 350 responses, it was necessary to avoid biasing estimates of the minima with RTs that may have been only occasional short-latency responses. Each subject's capability for rapid response can be estimated conservatively from a string of consecutive responses during the experimental session where both variability and response latency were minimal. Hence, the fastest responses of a chosen subset are less likely to be spurious if associated temporally with similarly fast responses. Moreover, the variable influence of factors such as motivation, attention, fatigue, etc., can be minimized with this procedure.

Guided by the above rationale, from each session we selected the string of 100 consecutive responses in which the sum of the interquartile range (the range of the middle $50 \%$ of the latency-ordered data) and Q1 was lowest. The histograms for these 100 consecutive responses are shown as the solid portion of the total response histograms (Fig. 3). As indicated by the arrows in Figure 3, for each solid histogram a defensible estimate of voluntary response capabilities should be in the range

\footnotetext{
${ }^{3}$ Given the evidence against successful anticipation of the stimulus, and the absence of reflex and startle responses, highly contiguous responses in the left tail of each response latency histogram should represent true stimulus-elicited RT responses. Spurious responses and false starts should be emitted with no pattern of onset latencies. For each session in the three experiments, three or fewer responses had a latency from 0 to $40 \mathrm{msec}$. As expected, inspection of the response latency histograms showed no contiguity for these unusually fast responses. Therefore, "outliers" were determined and rejected by examining each histogram for an interval in which no response occurred for a 4 -msec interval or more, beginning at the distribution median value and proceeding to shorter latencies. All responses to the left (faster) of the first such interval were eliminated. This procedure was extremely conservative considering the few responses that occurred prior to 40 msec latency. For all experiments $0.67 \%$ (27 of 4020 ) of the responses were eliminated, which indicates the sharpness of the left tails of the response latency histograms.
}

from the minimal latency to the $\mathrm{Q} 1$ value. As shown, the RT minima were 54.3 to $57.5 \mathrm{msec}$, and the $\mathrm{Q} 1$ values were 66.8 to $78.6 \mathrm{msec}$. For each subject, differences in the minima and Q1 values between the total and consecutive-response histograms were slight. S4 participated in three additional labial response sessions and showed an RT minimum of $46 \mathrm{msec}$, with a Q1 of 69 msec.

The earliest labial muscle response observed was a reduction of ongoing background OOI EMG. As shown in Figure 4, this reduced muscle activity was 10 to 30 msec in duration and was contiguous with the onset of phasic muscle activation associated with the voluntary lip elevation. Hence, the reductions observed had onset latencies 10 to $30 \mathrm{msec}$ shorter than latencies of muscle activations. The fastest reduction response had a latency of $35 \mathrm{msec}$. However, although reductions were observed for all subjects, including those in pilot experiments, the frequency of these reductions for any subject varied from 5 to $50 \%$ of the total responses. The enforced muscle relaxation often precluded observation of any reduction which may have been present. Reductions also were observed for the spontaneously initiated control responses (Fig. 5).

Experiment 2: Labial stimulus, lingual response. The results from this second experiment were subjected to the same scrutiny as in experiment 1 , regarding reflex responses, false starts, and elimination of outliers. As for experiment 1 , there was no evidence for reflex or startle responses in SL EMG in either the "do not respond" or the respond conditions. False starts were fewer than $0.9 \%$ (10 of 1165 total responses), and 9 responses were eliminated (see Footnote 3), of which only 3 were greater than $40 \mathrm{msec}$ in latency.

Response latency histograms are shown for each subject in Figure 6, following the same conventions as for experiment 1 (Fig. 3). Intermovement RT minima were from 52.0 to $59.0 \mathrm{msec}$, and Q1s were from 65.2 to 78.7 msec for the consecutive-response histograms. The two distributions formed by combining the consecutive-response histograms from experiments 1 and 2 across subjects (resulting in 400 responses in each composite distribution) are shown in Figure 7. As can be seen, the minima (54.3 and $52.0 \mathrm{msec}$ ) and Q1 values (69.9 and $73.3 \mathrm{msec}$ ) for the labial and lingual responses, respectively, are quite comparable. Thus, the obvious neural pathway differences for labial versus lingual responses to a labial stimulus appear to contribute insignificantly to the minimal response latencies.

Experiment 3: Auditory stimulus, labial response. The number of false starts (10 of 1426 responses) and responses less than $40 \mathrm{msec}$ in latency (3 of 1416 responses) were consistent with the previous two experiments. Eight responses greater than $40 \mathrm{msec}$ in latency were eliminated by the procedures described for experiments 1 and 2 (see Footnote 3 ). Acoustic startle responses were not evident in OOI EMG.

As for the previous two experiments, response latency histograms are shown for each subject in Figure 8 . As can be seen, RT minima and Q1s were from 56.7 to 62.9 msec and 71.6 to $81.5 \mathrm{msec}$, respectively. The RT minima to an auditory stimulus for $\mathrm{S} 2, \mathrm{~S} 3$, and $\mathrm{S} 4$ are 5 to 7 msec longer in latency than the RT minima for the same 


\section{LABIAL RESPONSE}


OOI EMG REACTION TIME (ms)-LABIAL STIMULUS

Figure 3. Response latency histograms (2-msec bin) of labial (OOI) muscle RTs from S1 to S4, experiment 1 (i.e., labial displacement stimulus). For each subject, the unshaded distributions include all responses after elimination of outlicrs (sce Footnote 3). Subject identification $(S)$ and the number of responses $(N)$ comprising each histogram are indicated. The solid histogram within each total response histogram is comprised of the 100 consecutive responses selected to represent optimal performance (see "Results"). For each solid histogram the RT minimum $(M)$ and the first quartile value $(Q 1)$ are indicated both numerically and with arrows along the abscissa.

subjects to a kinesthetic stimulus (experiment 1). The $\mathrm{Q} 1$ values for the auditory stimulus condition for $\mathrm{S} 1$ and S2 also show much longer latencies than for the kinesthetic stimulus.

\section{Discussion}

The present investigation was conducted to provide an estimate of RT minima in human orofacial muscles to kinesthetic stimuli as a basis for assessing the capability for contributions by RT processes to speech movement coordination. The RT minima of 46 to $57 \mathrm{msec}$ that were obtained are consistent with this hypothesis, particularly in that intermovement and intramovement RT minima were equivalent; short-latency RTs to displacement stimuli are not limited to autogenic actions. The RT minima to the auditory stimulus ( 57 to $63 \mathrm{msec}$ ), although longer in latency than those for the kinesthetic stimulus, sup- port earlier reports of rapid RTs to auditory stimuli in muscles of the finger (Luschei et al., 1967) and face (McFarlane, 1975).

The interpretation that RT processes may be involved in speech movement coordination is based partially upon the observations that these RTs overlap the range of latencies for intermovement muscle responses ( 25 to 80 $\mathrm{msec}$ ) that have been reported when labial speech movements were unexpectedly disturbed (Gracco and Abbs, $1982 \mathrm{~b}$ ). It is possible that RT processes operating naturally in coordination may be capable of shorter latencies than those observed here, if factors like stimulus-response compatibility, experience, and/or the stimulus uncertainty inherent to laboratory RT tasks are optimized. However, Gracco and Abbs (1982b) concluded that multiple sensorimotor processes contribute to the latency range that was observed. One subset of responses 


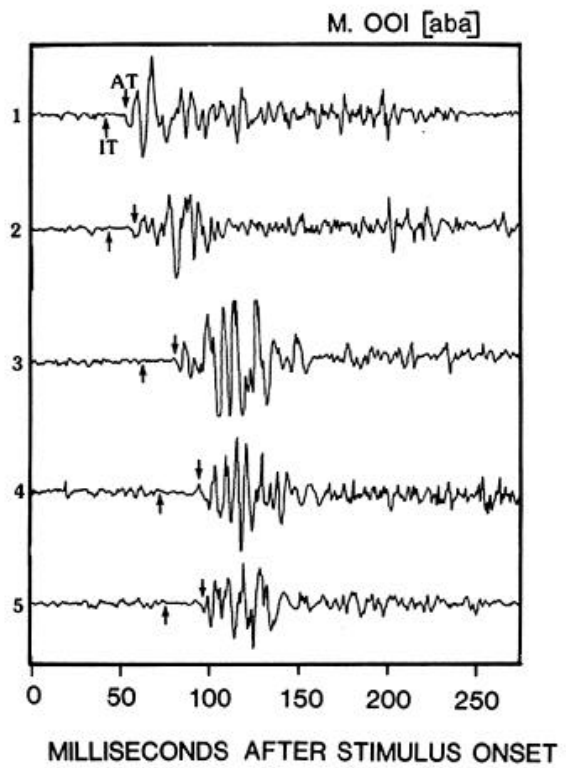

Figure 4. Labial (OOI) EMG for five RT responses from experiment 1 that show EMG reduction prior to the activation muscle response. For each record, the arrows indicate the reduction RT $(I T)$ and the activation EMG RT $(A T)$.

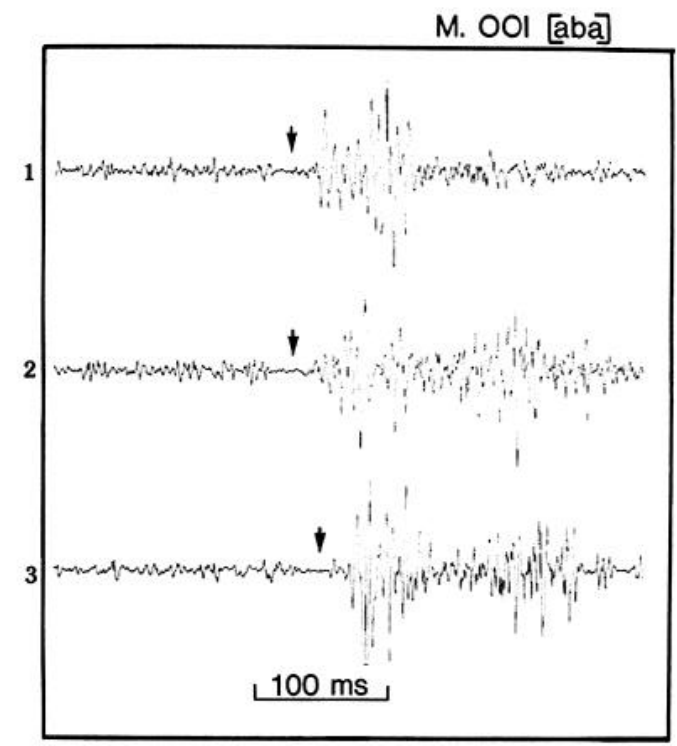

Figure 5. Labial (OOI) EMG for three spontaneous productions of the sound "aba." The arrows indicate periods of EMG reduction just prior to EMG activation.

ranged in latency from $50 \mathrm{msec}$ to more than $80 \mathrm{msec}$ and, thus, closely corresponds to the RT minima observed in the present experiments.

In addition to this latency criterion, the adaptive nature of RT processes appears well suited to explain some of the characteristics of the sensorimotor actions observed when speech movements were disturbed. The significant latency variation of these compensatory responses is consistent with a potential RT mechanism. Notably, the perioral reflex was uniformly absent, as in the present study. The compensatory response latencies varied due to an apparent dependence upon the timing of the load disturbance relative to the onset of the voluntary (requested) movement (Gracco and Abbs, $1982 b$ ); progressively shorter latencies were observed for loads delivered nearer in time to, and into the onset of, the agonist muscle burst for the voluntary movement. The compensatory muscle responses also were not fixed in latency relative to the onset of the agonist muscle burst. For loads delivered late in this agonist muscle burst, there was no compensatory response. The presence or absence of a particular compensatory response was also task specific. For example, labial muscle compensatory responses to jaw loads were seen only for speech tasks with combined lip-jaw goals, and are absent for jaw loads during speech tasks involving tongue-jaw goals (Kelso et al., 1982).

Sensorimotor actions with characteristics similar to those described in studies of speech movement perturbation have been reported in studies of automatic responses to postural disturbances (Nashner et al., 1979; Marsden et al., 1981; Nashner and Cordo, 1981; Cordo and Nashner, 1982). These rapid postural responses are also task specific, occur in muscles distant from the site of the disturbance, and show significant variation in latency. Nashner and Cordo (1981) noted that the stimulus-response characteristics of these adjustments indicate a more complex signal processing than for commonly studied reflexes. Similar to the results of the present experiments, Nashner and Cordo (1981) found that the latencies for RT movements to postural disturbance stimuli were as short as the latencies for automatic postural adjustments ( 80 to $120 \mathrm{msec}$ ), provided the former were well practiced and presented in a simple (no choice) context. Although unequivocal data are lacking, the major difference between RT processes and automatic speech and postural adjustments may be their functional objectives (cf. Nashner and Cordo, 1981) and the more extensive learning associated with the automatic adjustments. These postural data support the hypothesis that overlearned "RT processes" contribute to the coordination of complex movements.

In contrast to earlier arguments, the potential contribution of RT processes to speech coordination is also supported by preliminary analyses of multiple speech movement patterns (Abbs and Müller, 1980; Cole and Abbs, 1981; Abbs and Cole, 1982). In particular, Abbs and Cole (1982) noted that most classic discussions of afferent contributions to the coordination of movements were based on analyses of movement patterns under the implicit assumption that all relevant proprioceptive information is generated by the movement itself (e.g., Lashley, 1951; Kozhevnikov and Chistovich, 1965; Ohala, 1970; Kent and Moll, 1975; Kent, 1976). Thus, in attempts to discern requisite sensorimotor latencies for intermovement coordination, these latter investigators examined intervals among sequential and overlapping movements based on temporal measures of various movement parameters (e.g., movement onset, movement completion). However, as noted by Abbs and Cole (1982), the timing of afferent discharge associated with motor actions is not discernible from movement alone. For example, discharge from muscle spindles and cutaneous mechanoreceptors has been observed to be associated closely with the onset of muscle contraction and, thus, 


\section{LINGUAL RESPONSE}
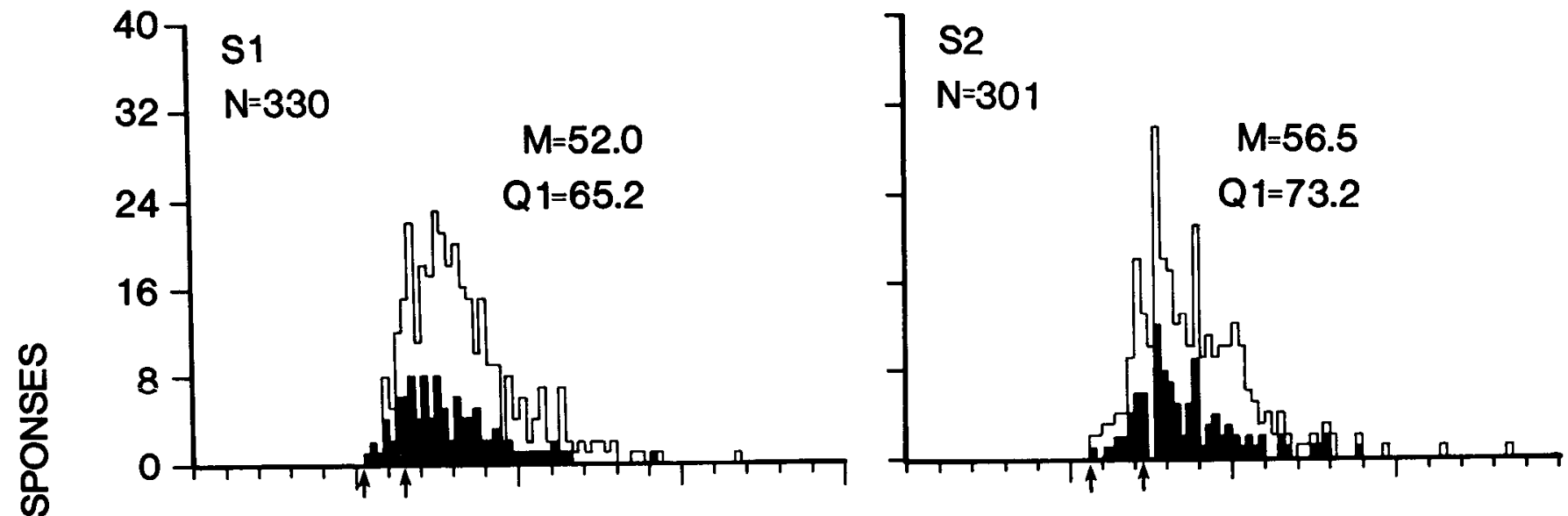

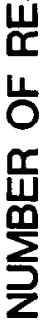
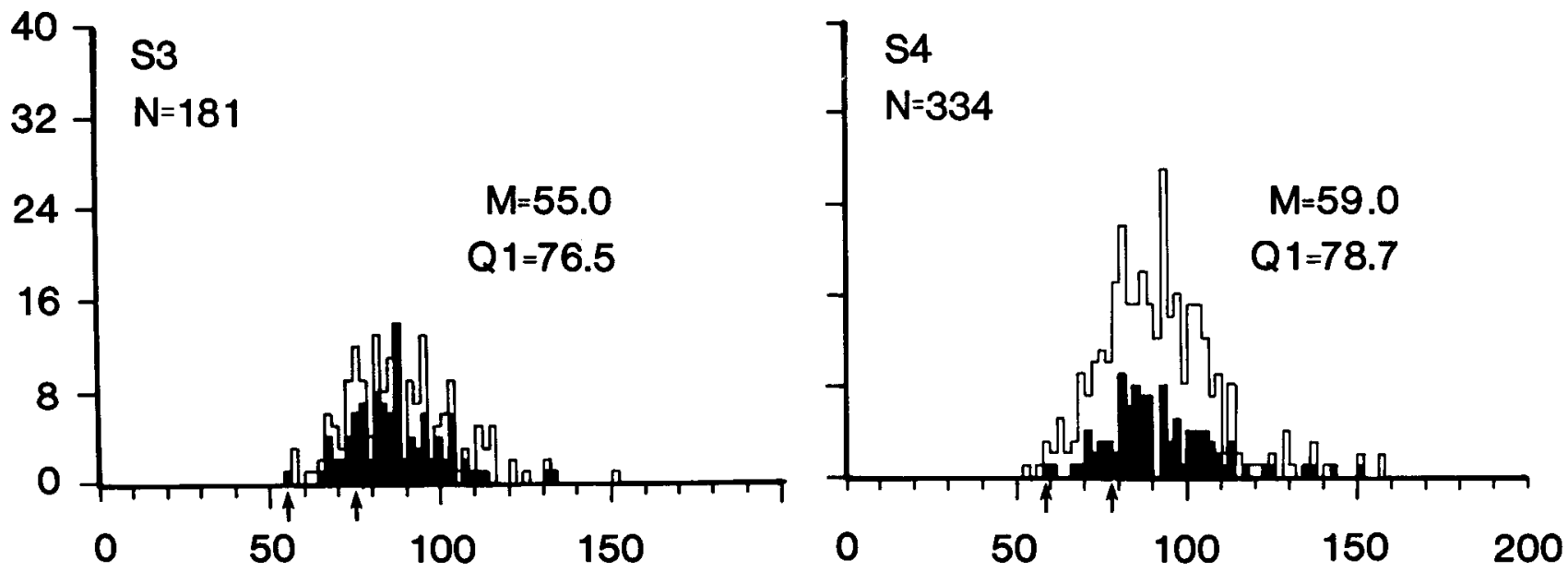

\section{SL EMG REACTION TIME (ms)- LABIAL STIMULUS}

Figure 6. Response latency histograms of lingual (SL) RTs from experiment 2 (i.e., lingual response to labial displacement stimulus). For each subject, the unshaded distributions include all responses after elimination of outliers (see Footnote 3). Subject identification $(S)$ and the number of responses $(N)$ comprising each histogram are indicated. The solid histogram within each total response histogram is comprised of the 100 consecutive responses selected to represent optimal performance (see "Results"). For each solid histogram the RT minimum $(M)$ and the first quartile value $(Q 1)$ are indicated both numerically and with arrows along the abscissa.

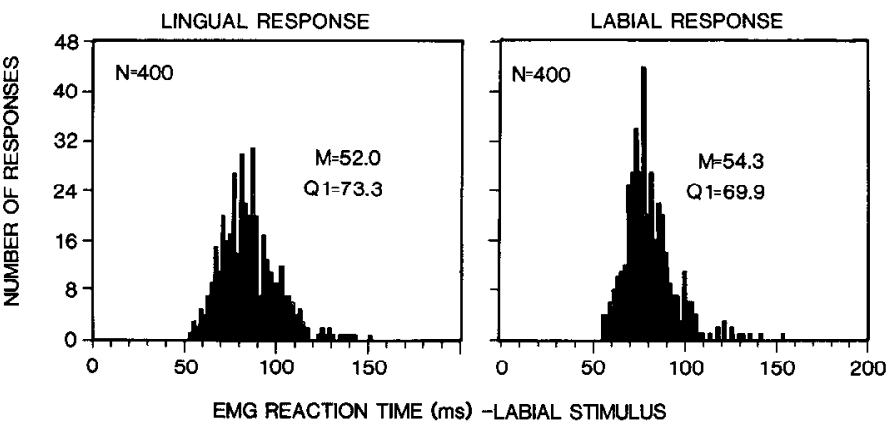

Figure 7. Composite response latency histograms of the 100 consecutive response histograms selected for each of the four subjects in experiment 1 (labial response, right panel) and experiment 2 (lingual response, left panel). The minimum $(M)$ and first quartile $(Q 1)$ values are indicated. precedes movement onset by varying magnitudes, depending upon the movements and muscles involved (Vallbo, 1973; Goodwin and Luschei, 1975; Hulliger et al., 1979). Based upon these considerations, preliminary analyses of movement and muscle activity patterns revealed that as much as 50 to $80 \mathrm{msec}$ may be available for sensorimotor actions among many lip-jaw and liptongue speech movements (Cole and Abbs, 1981; Abbs and Cole, 1982). These estimates are within the range of RTs observed in the present study.

Absence of reflexes. One benefit of investigating RT responses to a kinesthetic stimulus in the orofacial region was the absence of reflex contamination of the RT responses. Reflex activity has often confounded measurement of the minimal latency for volitional activity of the limbs and has necessitated the use of novel measurement 


\section{LABIAL RESPONSE}
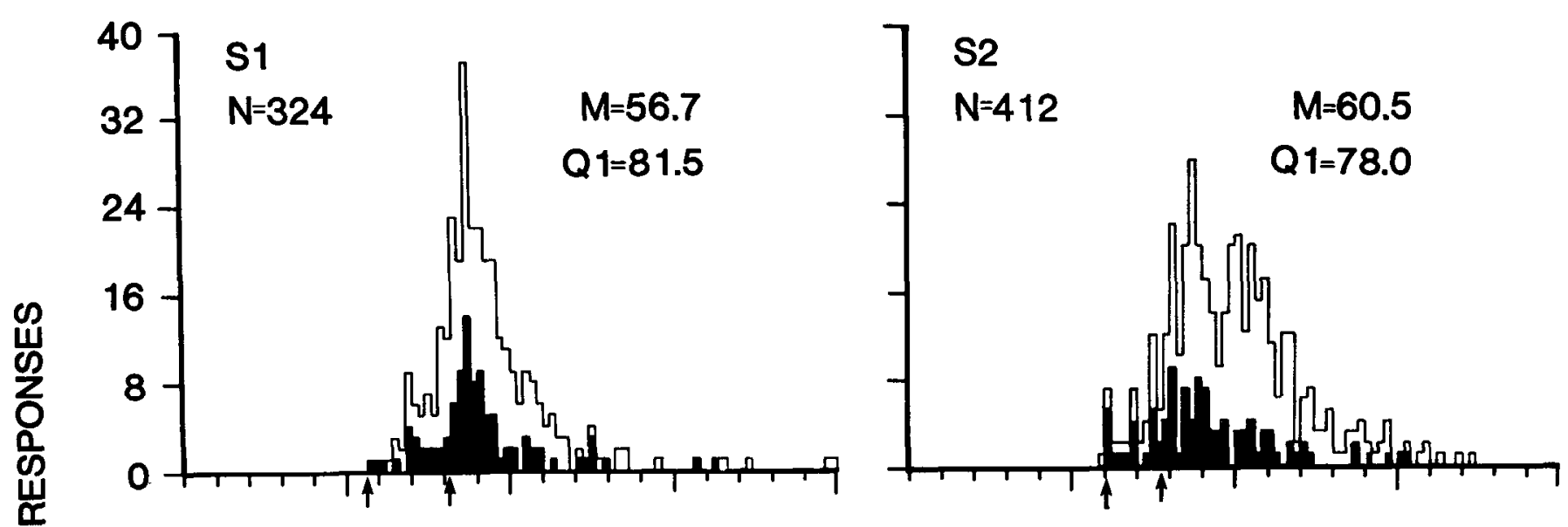

岁
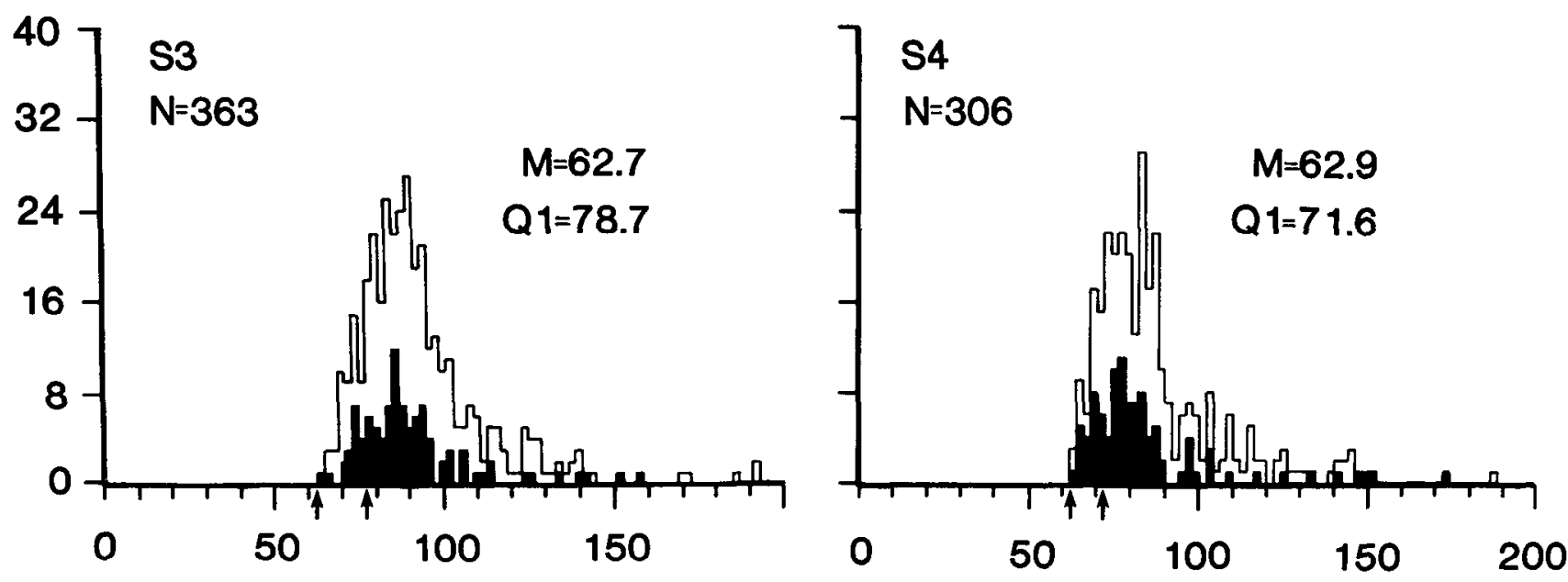

\section{OOI EMG REACTION TIME (ms) - AUDITORY STIMULUS}

Figure 8. Response latency histograms of labial (OOI) RT's from experiment 3 (i.e., labial response to the auditory stimulus). For each subject, the unshaded distributions include all responses after elimination of outliers (see Footnote 3 ). Subject identification $(S)$ and the number of responses $(N)$ comprising each histogram are indicated. The solid histogram within each total response histogram is comprised of the 100 consecutive responses selected to represent optimal performance (see "Results'). For each solid histogram the RT minimum $(M)$ and the first quartile value $(Q 1)$ are indicated both numerically and with arrows along the abscissa.

techniques (Crago et al., 1976) or special experimental procedures (Evarts and Granit, 1976). Consistent with the absence of reflex responses, the RT minima reported in the present study are longer in latency than any components of the perioral reflex. Also, the observed latency variability is characteristic of RT processes rather than reflexes. The failure of the labial stretch stimulus to evoke perioral reflexes is consistent with recent observations (Neilson et al., 1979; Gracco and Abbs, 1982b); skin taps or electrical stimuli have been reported to evoke perioral reflexes more readily than muscle stretch (Bratzlavsky, 1979; cf. Abbs and Cole, 1982). Thus, after careful examination of more than 7000 response records in these and pilot experiments, the most plausible interpretation of the measured latencies is that they represent valid latencies of the voluntary responses to the labial displacement signals; that is, RTs. In this regard, the RT minima observed in all three experiments are consistent with other reported RT minima (compare the introduction).

Reductions of background EMG. Response-related reductions in EMG activity in experiments 1 and 3 were the earliest responses to the stimuli and occurred at latencies as short as $35 \mathrm{msec}$. The consistent temporal juxtaposition of this EMG reduction with the subsequent activation indicates that the reduction is a component of the voluntary response (Yabe, 1975; Cole and Barlow, 1982). A similar reduction, termed premotion silence, has been reported for agonist limb muscles in the initiation of rapid voluntary movements (Gatev, 1972; Yabe, 1975, 1976; Mortimer and Eisenberg, 1982), and may be a separate phenomenon from the antagonist limb muscle suppression reported by Hufschmidt and Hufschmidt (1954). The variable appearance of the EMG reduction observed in the present experiments, both within and across subjects, is consistent with reports in limb mus- 
cles, perhaps reflecting the relation of this phenomenon to intended movement dynamics. Thus, the reduction has been reported to co-occur with more step-like EMG bursts (Cole and Barlow, 1982) and greater movement accelerations (Yabe, 1975; Mortimer and Eisenberg, 1982). Another potential function of premotion silence may be to transiently gate out afferent signals impinging upon the lower motoneuron pool at the initiation of rapid movements (cf. Bonnet and Requin, 1982; Cole and Barlow, 1982). The potential contributions to coordination by these very short-latency EMG reduction responses are not clear. However, because these responses have not been observed apart from their association with a subsequent voluntary activation, it would appear inappropriate to consider this reduction response as indicative of a general capability for short-latency suppression of muscle activity.

Validity of simple $R T$ paradigm. Consideration of the validity of using $\mathrm{RT}$ data from a simple (no choice) task as a basis for estimating potential naturally occurring parallel sensorimotor processes appears warranted. As a result of the learning that is involved in skilled, practical motor behaviors, the uncertainties as to the time of onset and the nature of upcoming afferent signals that are inherent to RT tasks would be reduced, as would be the response selection uncertainty in choice RT tasks. Also, as noted by Ghez (1979), transfer functions relating early sensory information to required output can be learned, with the ability to implement motor responses at short latencies. Thus, RTs in general, and choice RTs in particular, would seem to provide overly conservative estimates of the latency capabilities of the intentionally mediated sensorimotor processes that may be available for movement coordination. This perspective is essentially opposite that of some earlier theorists (e.g., Chernikoff and Taylor, 1952).

Potential contributions of RTs: Learned, predictive control. A rapid RT (intentionally mediated) process incorporated into control of ongoing movement would constitute an open-loop, afferent-dependent control mechanism. In open-loop, afferent-dependent control, afferent information is utilized in generating an appropriate motor action; the sensory contingencies and the sensorimotor input/output relations are hypothesized to be established, refined, and maintained with learning (Miles and Evarts, 1979; Houk and Rymer, 1981). Hence, such control is predictive. Given the obvious dependence of open-loop processes upon experience and learning, sensorimotor actions that are intentionally mediated appear well suited. Calibration between afferent and efferent signals, along with refinement and maintenance, has been observed for precise, voluntary forelimb movements under apparent open-loop control (Ghez and Vicario, $1978 \mathrm{a}, \mathrm{b})$. These latter experiments are of particular interest for their demonstration that, through learning, very early vibrissae afferent discharge can aid in rapid production (50 msec onset) of precise forelimb movements and isometric responses in the cat. Apparently, relevant target parameters were derived from the early afferent discharge, allowing prediction of the required responses. As task conditions changed, for example, when viscous or elastic loads were introduced to the forearm, appropriate motor responses were adapted to the "old" afferent signals. These rapid responses indicate potentially overlearned RT processes subject to modification with learning as task demands change.

In certain respects, coordination of the multiple movements in speech would appear ideally suited for openloop, afferent-dependent control. First, as has been noted, speed of operation, a hallmark of open-loop control, is a necessary prerequisite in the speech motor system. Second, open-loop, afferent-dependent control would appear useful in controlling actions among multiple structures that must act synergistically. Motor equivalence, in the form of covariable contributions between movements of the lips and jaw over multiple repetitions of a task, is apparent in speech movements (Hasegawa et al., 1976; Hughes and Abbs, 1976). Also, as has been discussed above, compensatory responses following unanticipated load disturbances of speech movements are distributed among the synergistic movements; that is, they are spatially dissociated from the movement that was disturbed. Based upon this dissociation, open-loop mechanisms would seem to be involved in generating these responses. Third, calibration of potential openloop, sensorimotor processes in the adult orofacial speech system would appear to be facilitated based on system stability, consistency of the intrapersonal space, and the predictability of load requirements. Given these considerations and the observations of acceptably short-latency inter- and intramovement RT actions, it is appealing to posit a role for these particular open-loop processes in speech movement coordination.

\section{References}

Abbs, J. H., and K. J. Cole (1982) Consideration of bulbar and suprabulbar afferent influences upon speech motor coordination. In Speech Motor Control, S. Grillner, B. Lindblom, J. Lubker, and A. Persson, eds., pp. 159-186, Pergamon Press, New York.

Abbs, J. H., and V. L. Gracco (1982) Motor control of multimovement behaviors: Orofacial muscle responses to load perturbations of the lips during speech. Soc. Neurosci. Abstr. 8: 282.

Abbs, J. H., and E. M. Müller (1980) Neurophysiological and biomechanical factors in articulatory movement. Paper presented at the Conference on the Production of Speech, Austin, Texas.

Baumeister, A. A., and C. E. Joubert (1969) Interactive effects on reaction time of preparatory interval length and preparatory interval frequency. J. Exp. Psychol. 82: 393-395.

Bonnet, M., and J. Requin (1982) Long loop and spinal reflexes in man during preparation for intended directional hand movements. J. Neurosci. 2: 90-96.

Bratzlavsky, M. (1979) Feedback control of human lip muscle. Exp. Neurol. 65: 209-217.

Chernikoff, R., and F. V. Taylor (1952) Reaction time to kinesthetic stimulation resulting from sudden arm displacement. J. Exp. Psychol. 43: 1-8.

Cole, K. J., and J. H. Abbs (1979) Kinesthetic reaction times in the speech motor system. J. Acoust. Soc. Am. 66 (Suppl. 1): S11.

Cole, K. J., and J. H. Abbs (1981) Short-latency labial afferentto-lingual efferent intended responses: Potential contributions to the spatial-temporal coordination of speech movements. J. Acoust. Soc. Am. 70 (Suppl. 1): S78. 
Cole, K. J., and S. M. Barlow (1982) Premotion silence in rapid voluntary movements for speech: Implications for suprabulbar control mechanisms. J. Acoust. Soc. Am. 71 (Suppl. 1): S34.

Cole, K. J., R. A. Konopacki, and J. H. Abbs (1983) A miniature electrode for surface electromyography during speech. J. Acoust. Soc. Am., in press.

Cordo, P. J., and L. M. Nashner (1982) Properties of postural adjustments associated with rapid arm movement. J. Neurophysiol. 47: 287-302.

Crago, P. F., J. C. Houk, and 7. Hasan (1976) Regulatory actions of the human stretch reflex. J. Neurophysiol. 39: $925-$ 935.

Evarts, E. V., and R. Granit (1976) Relation of reflexes and intended movements. Prog. Brain Res. 44: 1-14.

Evarts, E. V., E. Bizzi, R. Burke, M. DeLong, and W. T. Thach, eds. (1971) Central Control of Movement. Neurosci. Res. Program Bull., Vol. 9.

Folkins, J. W., and J. H. Abbs (1975) Lip and jaw motor control during speech: Responses to resistive loading of the jaw. J. Speech Hear. Res. 18: 207-220.

Folkins, J. W., and J. H. Abbs (1976) Additional observations on responses to resistive loading of the jaw. J. Speech Hear. Res. 19: 820-821.

Gatev, V. (1972) Role of inhibition in the development of motor coordination in early childhood. Dev. Med. Child Neurol. 14: $336-341$.

Ghez, C. (1979) Contributions of central programs to rapid limb movement in the cat. In Integration in the Nervous System, H. Asanuma and V. J. Wilson, eds., pp. 305-320, IgakuShoin, Tokyo.

Ghez, C., and D. Vicario (1978a) The control of rapid limb movement in the cat. I. Response latency. Exp. Brain Res. 33: $173-189$.

Ghez, C., and D. Vicario (1978b) The control of rapid limb movement in the cat II. Scaling of isometric force adjustments. Exp. Brain Res. 33: 191-202.

Goodwin, G. M., and E. S. Luschei (1975) Discharge of spindle afferents from jaw closing muscles during chewing in alert monkeys. J. Neurophysiol. 38: 560-571.

Gracco, V. L., and J. H. Abbs (1982a) Compensatory response capabilities of the labial system in relation to variation in the onset of unanticipated loads. J. Acoust. Soc. Am. 71 (Suppl. 1): S34.

Gracco, V. L., and J. H. Abbs (1982b) Temporal response characteristics of the perioral system to load perturbations. Soc. Neurosci. Abstr. 8: 282.

Hasegawa, A., M. J. McCutcheon, M. B. Wolf, and S. G. Fletcher (1976) Lip and jaw coordination during the production of $/ \mathrm{f}, \mathrm{v} /$. J. Acoust. Soc. Am. 59 (Suppl. 1): S85.

Hixon, T. J., M. D. Goldman, and J. Mead (1973) Kinematics of the chest wall during speech production: Volume displacements of the rib cage, abdomen, and lung. J. Speech Hear. Res. 16: 78-115.

Houk, J. C., and W. Rymer (1981) Neural control of muscle length and tension. In Handbook of Physiology, V. B. Brooks, ed., Section 1, Vol. II, Part 1, pp. 257-323, American Physiological Society, Bethesda, MD.

Hufschmidt, H. J., and T. Hufschmidt (1954) Antagonist inhibition as the earliest sign of a sensory-motor reaction. Nature 174: 607.

Hufschmidt, H. J., N. Kilimov, and D. Linke (1977) A very short reaction time in man. Electroencephalogr. Clin. Neurophysiol. 43: 622 .

Hughes, O., and J. H. Abbs (1976) Labial-mandibular coordination in the production of speech: Implications for the operation of motor equivalence. Phonetica 44: 199--221.

Hulliger, M., E. Nordh, A. E. Thelin, and A. B. Vallbo (1979) The responses of afferent fibres from the glabrous skin of the hand during voluntary finger movements in man. J. Physiol. (Lond.) 291: 223-249.

Hunker, C. J., and J. H. Abbs (1982) Respiratory movement control during speech: Evidence for motor equivalence. Soc. Neurosci. Abstr. 8: 946.

Kelso, J. A. S., B. Tuller, and C. A. Fowler (1982) The functional specificity of articulatory control and coordination. J. Acoust. Soc. Am. 72 (Suppl. 1): S103.

Kent, R. D. (1976) Models of speech production. In Contemporary Issues in Experimental Phonetics, N. J. Lass, ed., pp. 79-104, Academic Press, Inc., New York

Kent, R. D., and K. L. Moll (1975) Articulatory timing in selected consonant sequences. Brain Lang. 2: 301-323.

Kozhevnikov, V. A., and L. A. Chistovich (1965) Speech: Articulation and Perception, translation, Joint Publications Service, Washington D. C.

Lansing, R. W., and L. Meyerink (1981) Load compensating responses of human abdominal muscles. J. Physiol. (Lond.) 320: 253-268.

Lashley, K. S. (1951) The problem of serial order in behavior. In Cerebral Mechanisms in Behavior, L. A. Jeffress, ed., pp. 112-136, John Wiley \& Sons, Inc., New York.

Linke, D. (1977) Electromyography of the tongue. Electroencephalogr. Clin. Neurophysiol. 43: 621.

Luschei, E. S., C. Saslow, and M. Glickstein (1967) Muscle potentials in reaction time. Exp. Neurol. 18: 429-442.

MacNeilage, P. F. (1970) Motor control of serial ordering of speech. Psychol. Rev. 77: 182-196.

Marsden, C. D., P. A. Merton, and II. B. Morton (1981) IIuman postural responses. Brain 104: 513-534.

McFarlane, S. M. (1975) Neural response time of stutterers and nonstutterers for certain oral motor tasks as a function of auditory stimulation. Doctoral dissertation, University of Washington, Seattle.

Miles, F. A., and E. V. Evarts (1979) Concepts of motor organization. Annu. Rev. Psychol. 30: 327-362.

Mortimer, J., and P. Eisenberg (1982) Pre-movement silence in agonist muscles preceding maximum power arm movements. Soc. Neurosci. Abstr. 8: 730.

Nashner, L. M., and P. J. Cordo (1981) Relation of automatic postural responses and reaction-time voluntary movements of human leg muscles. Exp. Brain Res. 43: 395-405.

Nashner, L. M., M. Woolacott, and G. Tuma (1979) Organization of rapid responses to postural and locomotor-like perturbation of standing man. Exp. Brain Res. 36: 463-476.

Neilson, P. D., G. Andrews, B. E. Guitar, and P. T. Quinn (1979) Tonic stretch reflexes in lip, tongue, and jaw muscles. Brain Res. 178: 311-327.

Ohala, J. (1970) Aspects of the control and production of speech. Working Papers in Phonetics, University of California 15.

Schupp, W., and C. Schlier (1972) The dependence of simple reaction time on temporal patterns of stimuli. Kybernetik 11: 105-111.

Vallbo, A. B. (1973) Muscle spindle afferent discharge from resting and contracting muscles in normal human subjects. Prog. Clin. Neurophysiol. 3: 251-262.

Weiss, P. (1941) Does sensory control play a constructive role in the development of motor coordination? Schweiz. Med. Wochenschr. 12: 406-407.

Wyrick, W., and A. Duncan (1974) Electromyographical study of reflex pre-motor and simple reaction time of relaxed muscle to joint displacement. J. Motor Behav. 6: 1-10.

Yabe, K. (1975) Electromyographic silent period preceding a rapid voluntary movement. In Proceedings of the Fifth International Congress of Biomechanics, P. V. Komi, ed., pp. 7581, University Park Press, Baltimore.

Yabe, K. (1976) Premotion silent period in rapid voluntary movement. J. Appl. Physiol. 41: 470-473. 